\title{
Librational motion of $\mathrm{CO}$ in solid Ar: Raman and IR spectra and quantum simulations
}

\author{
Johan Lindgren ${ }^{1}$, Adriana Olbert-Majkut ${ }^{2}$, Mika Pettersson ${ }^{1}$, and Toni Kiljunen ${ }^{1}$ \\ ${ }^{I}$ Department of Chemistry, Nanoscience Center, P.O. Box 35, FI-40014 University of Jyväskylä, Finland \\ E-mail: toni.k.kiljunen@jyu.fi \\ ${ }^{2}$ Faculty of Chemistry, Wroclaw University, F. Joliot-Curie 14, 50-383 Wroclaw, Poland
}

Received April 20, 2012

\begin{abstract}
Rovibrational Raman spectra of CO molecules isolated in solid Ar were measured for the 9-30 K temperature range and compared to past and present IR spectra. The fundamental band appears as a triplet-split structure, where the center peak shows completely different response to temperature in the Raman and IR spectroscopies. The peak is sharp and stable in Raman but reversibly broadens beyond recognition in IR upon annealing. The red-shifted, intense line of the triplet is found thermally inert similarly in both spectroscopies. The third line is the weakest, and as concentration dependent, it is assigned to a dimer as before. The $\mathrm{CO}-\mathrm{H}_{2} \mathrm{O}$ impurity complex is identified as a side band. We employ crystal field and quantum chemical modeling to interpret the disparity between the spectroscopies. The stable and broadening lines are given assignments to double- and singlesubstitution sites, respectively. Thermal excitations are not effective in the former case of angularly tightconfined, deep potential well. In the single-substitutional case, the librational level structure shows up in discriminating between the Raman and IR selection rules. An effectively $\Delta J=0$ totally symmetric transition is found for Raman that is uncoupled from lattice phonons and corresponding broadening mechanisms. The low-temperature limit necessitates the use of a fixed lattice approach while the warmer end of the range is best described with an adiabatic, pseudorotating lattice approach.
\end{abstract}

PACS: $33.20 . \mathrm{Ea}$ Infrared spectra ;

33.20.Fb Raman and Rayleigh spectra (including optical scattering);

33.20.Vq Vibration-rotation analysis;

34.20.Gj Intermolecular and atom-molecule potentials and forces;

31.15.xv Molecular dynamics and other numerical methods.

Keywords: matrix isolation spectroscopy, solid argon, $\mathrm{CO}$ molecule, rovibrational analysis, hindered rotation, libration-phonon interaction, crystal field theory, $a b$ initio calculation.

\section{Introduction}

Atomic and molecular impurities in solids can be optically probed to interrogate the local structure and energetics of the relaxed surroundings as well as the dynamical interplay between the impurity and host. Motional degrees of freedom within the trapping cage are reflected in the spectral widths and intensity patterns of Raman and IR spectroscopies. An example of the latter is given by Khriachtchev et al. [1] for the $\mathrm{HXeBr}$ and $\mathrm{HKrCl}$ molecules, where the spectral fingerprints manifest different site effects and librations. In the present work, we report and analyze our spectroscopic results regarding the matrixisolated CO molecule, which was obtained as purely deposited component of the gas mixture sprayed on the cold substrate. Using computational modeling, we discuss the Raman spectroscopic observations in terms of rotational motion to quantify the degree of hindrance, and compare to previous and present results obtained by IR spectroscopy.

The concept of hindered rotation of molecules in crystals, and the corresponding determination of potential energy by means of Raman effect or infrared measurements, can be traced back to Pauling [2]. The study of vibrational and rotational degrees of freedom of $\mathrm{CO}$ molecule in crystalline solids has been quite comprehensive, and a recent, vast collection of spectroscopic studies and explaining theories was compiled by Fajardo et al. [3] In particular, the solid argon case has received considerable attention [4-17]. (For the gas phase Raman case, see Thibault et al. [18].) The debate over the assignment of the observed transition 
bands lasted over three decades. Although the main focus was on the question whether some of the spectral bands should be assigned to $\mathrm{CO}$ monomer or dimer, some earliest studies $[6,7,11]$ suggested that there were no rotational motion at all. Latest studies [15-17] concluded, in agreement with most of the previous results [5,8-10], that the observed IR spectrum indeed shows signs of rotations based on the reversible temperature dependence of the band widths. In addition to differences in sample preparation and detection setups, the discrepancy between the past and present relates to the increased spectral resolution. Line broadening processes inherent in rare gas solids further challenge the interpretations based on a delicate effect. The question remained, therefore, to which extent is the rotational motion coupled to lattice vibrations.

The series of experiments by Abe et al. [15-17,19] showed that there are two distinct absorption bands resulting from monomeric $\mathrm{CO}$ with different frequencies, shapes and temperature dependencies. The red-shifted one nominated as "A" results from a stable site, where rotations are quenched, while the site " $\mathrm{B}$ ", with the band origin $1.8 \mathrm{~cm}^{-1}$ to the blue, allows librational motion that couples with lattice oscillations. The line " $\mathrm{B}$ " therefore reversibly changes intensity upon annealing due to the librational energy dispersal. The site structure was not speculated further. Anderson and Winn [14] combined high-resolution IR with line shape theory to identify the local geometry of Ar atoms surrounding either native or photolytically from OCS produced CO molecules. In the former case, the band separation was again $1.8 \mathrm{~cm}^{-1}$, but their interpretation discards rotation. Instead, they elaborate on the center-ofmass translation of a directionally fixed $\mathrm{CO}$ to dictate the shape of the vibrational transitions. After relaxation, they treat the argon lattice fixed upon rotation of the molecule to obtain angular potentials. As a consequence, the crystal field effect is large and no librational splitting patterns for the ground level were generated. It was noted, however, that adiabatic relaxation with respect to molecular angle did not change the potential dramatically. Their assignment to two sites, apart from the translation-vibration analysis, was in qualitative agreement with Abe's interpretation for the native $\mathrm{CO}$. The two-site model resulted as an adjusted superposition of the so-called cluster ("A") and crystal ("B") configurations that they used for building the initial lattice. Both of these were double-substitution trapping sites, whereas the photoproduct $\mathrm{CO}$ exhibited a different, single-substitutional trapping. The cluster ("A") configuration was found translationally more localized than the crystal ("B") counterparts, which was concluded as the origin for the different temperature dependence [14].

Successful Raman measurements require a better optical quality from the samples. This can also be seen as the merit of the Raman, since lattice imperfections, inhomogeneities, and other such effects that can lead to a scrambled spectral outcome, must have been minimized. As far as we know, the Raman spectra of CO in solid Ar are reported here for the first time. The rovibrational band origin is an important marker of the local lattice geometry. Our finding that the lines are identically matrix-shifted in Raman and IR experiments conforms the good quality of the previous samples. Raman spectroscopy is complementary to the IR due to the same vibrational $v=0 \rightarrow 1$ origin here. However, the selection rules are different for the rotational part which should affect the spectral patterns. Here the spectral patterns that would manifest crystal-field splitting are hidden due to broadening mechanisms. Instead, the lines can be attributed to different sites. The present work builds on the remarkable new observation, where the peak " $\mathrm{B}$ " is found thermally stable in Raman. This is opposite to the IR result and necessitates an reappraisal of the previous theory. We shall present a theoretical analysis with regard to the emergence of peak patterns in both Raman and IR spectra due to electrostatic and thermal effects. The crystal field effect on rotational levels is shown for two trapping configurations; a single-substitution for " $\mathrm{B}$ " and a doublesubstitution for "A", where in the latter case a vacancy is introduced to a nearest neighbor site before optimizing the geometry of the simulation cube.

\section{Methods}

\subsection{Experimental methods}

Carbon monoxide (purity $99.997 \%$, Messer) was mixed with $\operatorname{argon}(99.999 \%$, Messer) in a vacuum line and stored in a 51 pyrex bulb prior to the matrix deposition. The $\mathrm{CO} / \mathrm{Ar}$ matrix ratio was set to either $1 / 150$ or $1 / 750$ by standard manometric techniques and dilutions.

The deposition temperature was $19 \mathrm{~K}$ to optimize the matrix quality. For the Raman measurements, the gas mixture was deposited onto a thin $(150 \mu \mathrm{m})$ sapphire substrate kept at $19 \mathrm{~K}$ in closed-cycle cryostat (APD Cryogenics) equipped with quartz windows. To obtain an optically clear matrix, the deposition rate was maintained below $0.11 \mathrm{mmol} / \mathrm{min}$ and the total amount of the deposited gas was about $12-40 \mathrm{mmol}$. The thickness of the matrix film was ca. $40 \mu \mathrm{m}$. The sample temperature was controlled with a Lakeshore 330 temperature controller equipped with a silicon diode.

Raman measurements were performed using the backscattering geometry as described previously [20]. The excitation source was a solid-state diode-pumped laser (Verdi, Coherent) operating at $532.0 \mathrm{~nm}$, and a power level up to $600 \mathrm{~mW}$ at the sample was used. The laser beam was focused on a sample with an achromatic lens of $12 \mathrm{~cm}$ focal length. The Rayleigh scattering was attenuated with an edge filter (Semrock, laser line blocking: OD >6). The Raman scattering from a sample was collected and focused on the spectrograph slit with an achromatic lens of $15 \mathrm{~cm}$ focal length. The slit width used for measurements was $40 \mu \mathrm{m}$. The Raman signal was dispersed with an imaging 
spectrograph of $50 \mathrm{~cm}$ focal length, equipped with 600 and 1200 grooves $/ \mathrm{mm}$ ruled gratings and a 2400 grooves $/ \mathrm{mm}$ holographic grating. The latter grating was used for recording the spectra presented in this paper. The $1 \mathrm{~cm}^{-1}$ resolution Raman spectra were recorded with a CCD camera (Andor Technology, Newton) mounted on the spectrograph (Acton Research Corporation, SpectraPro 2500i). Wavenumber calibration was performed using the rich rovibrational Raman line pattern of a gaseous CO sample as the internal standard, which is accurate to within $\pm 0.1 \mathrm{~cm}^{-1}$, and when mechanical repeatability in day-to-day alignment is taken into account, the absolute accuracy remains well within $\pm 1 \mathrm{~cm}^{-1}$ tolerance.

For IR measurements, the matrix was prepared similarly by deposition of the gaseous sample onto a CsI window kept at $19 \mathrm{~K}$. The low temperature was maintained by means of a closed-cycle helium refrigerator (ARS-2HW). FTIR spectra were recorded between $2400-2000 \mathrm{~cm}^{-1}$ in a transmission mode by means of a Bruker 66 FTIR spectrometer with a resolution of $0.25 \mathrm{~cm}^{-1}$ and using a liquid $\mathrm{N}_{2}$ cooled MCT detector. After the matrix deposition, the spectra in the entire MIR region (4000-400 $\mathrm{cm}^{-1}$ ) were also recorded with a resolution of $0.5 \mathrm{~cm}^{-1}$.

\subsection{Theoretical methods}

A guest molecule placed in a rare gas solid probes the electrostatic field generated by the surrounding host atoms. Two limiting cases arise when considering the rotational degree of freedom. One, where nearly free rotation emerges, and the other end, where rotation is reduced to a harmonic motion with molecular axis oscillating around some crystalline direction. The intermediate, hindered rotor regime, is best understood as rotational tunneling between the minima of the angular potential energy surface. Depending on the crystal field strength, the asymptotic freerotor states begin to coalesce and finally arrange into different manifolds of varying degrees of degeneracy. The resulting states are commonly referred to as librational states, in accord with the notion of vibration-like motion of the rotational degree of freedom [21].

Regarding the local symmetry, the problem of rotational motion of diatomics in solids has been approached from two limits. The perfect lattice limit neglects rotationphonon couplings but may include rotation-translation via eccentric motion. The first three-dimensional model for rotating diatomic molecule in an octahedral $\left(O_{h}\right)$ crystal field was developed by Devonshire [22]. This model and its extensions commonly referred to as crystal field theory use the inherent symmetry of the crystal to derive the potential used in a rotational Hamiltonian. The other extreme to solve the rotational potential is to lift the rigid cage approximation and to allow the center of mass of the guest molecule to fully relax into equilibrium position for each orientation. This procedure largely preserves the symmetry, and is termed as pseudorotation by Manz [23].
We will use the latter, adiabatic approach in our simulations. This assumption relies on the relatively slow rotational motion of $\mathrm{CO}$ with respect to longitudinal lattice phonon modes that can reach $2.0 \mathrm{THz}$ in Ar. In addition, we use a model where the lattice structure is optimized once for the known minimum energy orientation of $\mathrm{CO}$, but fixed for subsequent rotations. Numerical solution of the nuclear Schrödinger equation is then performed for these potential energy surfaces to obtain the librational eigenstates and energies. In the adiabatic, pseudorotation case, we use the molecule and Ar atom trajectories to determine the effective rotational constant of the $\mathrm{CO}$ molecule. Thus the simulation of the spectra is interconnected via the solution of time-independent Schrödinger equation to manually rotating the $\mathrm{CO}$ molecule in Ar crystal.

In the potential energy evaluations, the guest molecule is placed in a 256-site fcc simulation cube in a central single- or double-substitution position. In addition to the guest molecule, two surrounding shells of Ar atoms are allowed to relax to their equilibrium positions by minimizing the overall potential energy, which is a pairwise sum of molecule-atom and atom-atom potentials:

$$
V=\sum_{i<j} V_{\mathrm{Ar}_{i}, \mathrm{Ar}_{j}}+\sum_{i} V_{\mathrm{Ar}_{i}, \mathrm{CO}} .
$$

Here, the isotropic interaction between argon atoms is of Tang-Toennies type [24], and the potential between carbon monoxide and argon atom is taken from Pedersen et al. [25]. The molecular part is written as [25]

$$
V_{\mathrm{Ar}_{i}, \mathrm{CO}}=V_{\mathrm{CO}}(r)+V_{\mathrm{Ar}_{i}, \mathrm{CO}}\left(r, R_{i}, \theta_{i}\right),
$$

where $V_{\mathrm{CO}}(r)$ is the empirical vibrational potential energy curve of the isolated CO molecule [26] and $V_{\mathrm{Ar}, \mathrm{CO}}\left(r, R_{i}, \theta_{i}\right)$ is the three-dimensional $a b$ initio intermolecular potential energy surface. The variables $r, R_{i}$, and $\theta_{i}$ are the bond length of $\mathrm{CO}$, the distance from center of mass of $\mathrm{CO}$ to $i$ th argon atom, and the angle between position vectors $\mathbf{r}$ and $\mathbf{R}$. The position vector $\mathbf{r}$ points from $\mathrm{C}$ to $\mathrm{O}$ and $\mathbf{R}$ points from the center of mass of $\mathrm{CO}$ to argon. Optimized lattice was obtained using only the intermolecular part of Eq. (2) in the gradient descent. Finally, the potential is mapped in the form $V=V(\theta, \phi)$, where the polar and azimuthal angles represent the orientation of the molecule relative to the space-fixed (crystal) axes.

With the adiabatic or fixed three-dimensional potential energy surfaces at hand, the rotational states are obtained by diagonalization of the Hamiltonian matrix

$$
\begin{aligned}
& H_{j m, j^{\prime} m^{\prime}}=B_{\mathrm{eff}} j(j+1) \delta_{j j^{\prime}} \delta_{m m^{\prime}}+ \\
& +\int d \Omega Y_{j m}^{*}(\theta, \phi) V(\theta, \phi) Y_{j^{\prime} m^{\prime}}(\theta, \phi),
\end{aligned}
$$

with spherical harmonics (real form) as basis functions. Adopting the concept of pseudorotation, the effective moment of inertia increases due to the rotation of the molecule around the center of interaction located at the center 
of the cage, and due to the synchronously transforming cage [23]. With this model, the rotational constant then reduces to an estimated, effective one $B_{\text {eff }}$. The vibrational state $v=0,1$ dependence of the potential and rotational constant can be included by using $\mathrm{CO}$ bond lengths $r_{0}$ and $r_{1}$ when performing the adiabatic rotational potential evaluations. The evaluations were done on a coarse grid with a 5-degree spacing, and then spline-interpolated to final $[\theta, \phi]$ grid size of $180 \times 360$ points. Since the potential and the level structure do not change markedly when going from $r_{0}=1.1305 \AA$ to $r_{1}=1.1357 \AA$, we drop the $v$-dependence from the due discussion. Molecular trajectories in the gradient descent reveal translational motion amplitude from the cage center that ranges up to $0.26 \AA$ along the momentary fixed directions. The lattice atoms travel less than $0.08 \AA$ to the momentary equilibrium positions thus leading to $B_{\text {eff }}=1.24 \mathrm{~cm}^{-1}$ value for the singlesubstitutional case. In the double-substitutional case, the symmetric transformation of the cage is broken rendering the pseudorotation concept intractable. Moreover, the molecule is unlikely to rotate in this site. For these reasons we abandon the down-scaling from $B_{0}=1.92 \mathrm{~cm}^{-1}$ value.

Within the dipole approximation, molecule-light interaction Hamiltonian is

$$
\hat{H}_{\text {ind }}=-\hat{\mu} \cdot \varepsilon \text {. }
$$

The interaction Hamiltonian is defined with respect to space-fixed axes, which are defined by the crystal frame. Electric field defines the laboratory frame. We assume linearly polarized light, and choose the polarization direction as the laboratory $Z$ axis. The dipole moment of $\mathrm{CO}$ points in the direction of the symmetry axis of the molecule, and the direction of the dipole moment vector is chosen as the $z$ direction of the molecular frame. In the Raman effect, the interaction Hamiltonian is expressed as

$$
\hat{H}_{\text {ind }}=-(\alpha \cdot \varepsilon) \cdot \varepsilon .
$$

In the spherical basis this becomes

$$
\hat{H}_{\text {ind }}=-\sum_{\lambda, q}(-1)^{\lambda+q} \alpha_{-q,-\lambda} \varepsilon_{\lambda} \varepsilon_{q} .
$$

To express the polarizability tensor in molecular frame, it is easier to express it first in an irreducible basis using connection between coupled and uncoupled representations, i.e., $\quad \alpha_{-q,-\lambda}=\sum_{L, M}\langle 1-q 1-\lambda \mid L M\rangle \alpha_{M}^{(L)}$. We use the Wigner D-functions $D_{M m}^{(L)}(\theta, \phi, \chi)$, to relate the components $M$ in the space-fixed frame to $m$ in the molecule-fixed frame under a rotation $\theta, \phi, \chi$ [27]. In molecular frame, only components with $m=0, L=0$ and $m=0, L=2$ are nonzero [28]. The total intensity for a transition from an initial state $i$ to a final state $f$ is obtained as an average over possible crystal orientations

$$
I_{i \rightarrow f}=\frac{1}{8 \pi^{2}} \int d \Omega^{\prime}\left|\left\langle\Psi_{i}\left|\hat{H}_{\text {ind }}\right| \Psi_{f}\right\rangle\right|^{2},
$$

where the primed variables $\Omega^{\prime}=\theta^{\prime}, \phi^{\prime}, \chi^{\prime}$ are Euler angles between laser field (lab frame) and crystal frame. In the Raman process this reduces to

$$
I_{i \rightarrow f}=\frac{16 \pi \Delta \alpha^{2} \varepsilon^{4}}{15^{2}} \sum_{m=-2}^{2}\left|\left\langle\Psi_{i}\left|Y_{2 m}(\theta, \phi)\right| \Psi_{f}\right\rangle\right|^{2},
$$

where we have neglected the Rayleigh part and defined the anisotropy of polarizability $\Delta \alpha=\alpha_{\|}-\alpha_{\perp}$. The intensity of radiation in infrared spectrum is obtained the same way as above, but now the intensity is proportional to permanent dipole moment:

$$
I_{i \rightarrow f}=\frac{\mu_{v^{\prime}, v} \varepsilon^{2}}{5} \sum_{m=-1}^{1}\left|\left\langle\Psi_{i}\left|Y_{1 m}(\theta, \phi)\right| \Psi_{f}\right\rangle\right|^{2} .
$$

Here we have defined $\mu_{v^{\prime}, v}=\left\langle v^{\prime}\left|\mu_{0}\right| v\right\rangle$ as the transition dipole moment between vibrational states $v^{\prime}$ and $v$.

Two-dimensional Gaussian quadrature was used to compute the potential energy matrix elements and the transition intensities that require a numerical integration over the surface of the unit sphere [29]. The coefficients in above intensity equations were set to unity, and the intensity for a certain transition $i \rightarrow f$ was multiplied by the corresponding Boltzmann factor. We do not attempt to computationally define the level dependent line widths. Instead the broadening mechanisms are discussed based on previous knowledge on phonon couplings [10,30]. For visualization purposes, we include a Lorentzian waveform for transition frequencies with $\mathrm{FWHM}=1 \mathrm{~cm}^{-1}$. The $v=0 \rightarrow 1$ fundamental frequency (band origin) was calculated using the 3D potential energy surface in Eq. (2), summing over a sufficient number of nearest argon atoms, and solving for the vibrational states by a Fourier-GridHamiltonian method (FGH) [31]. The solution was made on a grid with 256 points, and in order to get converged energies, the bond length $r$ range from $0.9515 \AA$ to $1.3939 \AA$ was taken slightly wider than tested by the developers of the potential [25]. In Eq. (2), the variables $R$ and $\theta$ get their values from adiabatic potential energy evaluations.

We performed a set of ab initio calculations in order to check on the relative transition intensities and wavenumbers for single- or double-substitutional $\mathrm{CO}$ trapping cases. Twelve or eleven nearest-neighbor argons were included, respectively, such that the initial structure was that of the above obtained energy minimum and only $\mathrm{C}$ and $\mathrm{O}$ were further relaxed in order to get reasonable results. We used the RI-MP2/def2-TZVPP theory [32-35] within the Turbomole program package [36].

\section{Results}

\subsection{Raman and IR measurements}

We plot the IR absorption spectra in the upper panel of Fig. 1. The observable four distinct bands, labeled from A to $\mathrm{D}$, are previously attributed to A) a tight-site $\mathrm{CO}$, 

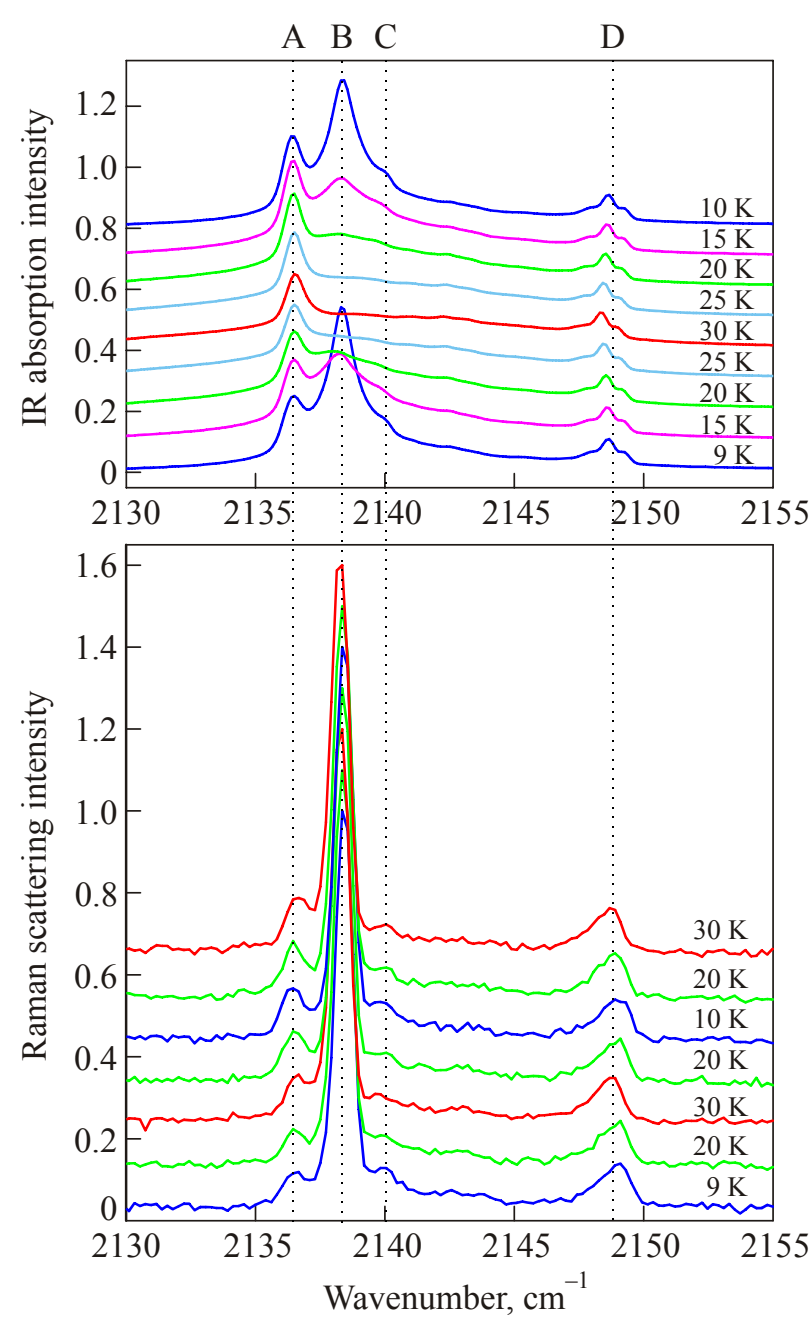

Fig. 1. (Color online) The fundamental region of the CO Raman (lower panel) and IR (upper panel) spectra in solid Ar for the 1/750 sample dilution. The bottom $9 \mathrm{~K}$ spectra are measured after the deposition, and the vertical order of plotting follows the subsequent annealing cycle that extends to $30 \mathrm{~K}$. Peak maxima are located at $2136.5,2138.3,2139.9$, and $2149 \mathrm{~cm}^{-1}$. See text for the A-D band assignments, respectively.

B) a loose-site $\mathrm{CO}, \mathrm{C}$ ) a $\mathrm{CO}$-dimer, and $\mathrm{D}$ ) a $\mathrm{CO}-\mathrm{H}_{2} \mathrm{O}$ complex. The temperature series also reproduces the previous observations, where the reversible band broadening occurs for the B labeled site (and possibly for C) but not for A (and D). Third agreement between the present and previous research result is the intensity ratio of the doublet $\mathrm{A}$ and $\mathrm{B}$ which seems to favor the $\mathrm{B}$ component at low temperature. Because the $10 \mathrm{~K}$ spectra after the annealing cycle are practically identical to those obtained directly after deposition and cooling down to $9 \mathrm{~K}$, all the bands can be considered to originate from stable site structures.

The Raman spectra in the lower panel of Fig. 1 accurately reproduce the A-D band locations. The spectrum appears rather like a triplet band shape with the main peak at $2138.3 \mathrm{~cm}^{-1}$ and pronounced shoulder peaks at 2136.5 and $2139.9 \mathrm{~cm}^{-1}$. The water complex is located at
$2149 \mathrm{~cm}^{-1}$ as in IR. The striking difference between the spectroscopies is, however, the behavior of the B-labeled feature that now remains intact despite the temperature cycling. This new information calls for a revision of the assignments.

In the more concentrated, 1/150 sample, the $\mathrm{A}$ and $\mathrm{C}$ peak intensities were comparable at $9 \mathrm{~K}$ temperature, while the $\mathrm{C}$ side of the Raman triplet becomes weaker at the shown $1 / 750$ dilution. It is a bit surprising to see the dimerassigned peak to surface at all with this dilution, since it disappeared already for $1 / 500$ in the IR measurements of Abe et al. [15] who, on the other hand, deposited at $8 \mathrm{~K}$. The $\mathrm{C}$ line tends to melt away at elevated temperatures, especially in the more concentrated sample. The D band assigned to a water complex is broad and asymmetric, and its intensity maximum exhibits a reversible drift along with the temperature. This side band was less pronounced in 1/150 dilution, since the amount of impurity $\mathrm{H}_{2} \mathrm{O}$ remains approximately constant [15].

\subsection{Single-substitutional case}

We begin with presenting the fixed and adiabatic potential energy surfaces in Fig. 2 to be used for the energy level and corresponding Raman and IR spectrum calculations. The former assumes a limit where the molecule never samples the high-energy part but is bound to a small-amplitude, fast oscillation around the minimum energy orientation. The latter limit corresponds to a thermally allowed sampling of the barrier, and its time-dependent response, in a process of rotational diffusion. In another aspect this can be seen as a tunneling regime as well. Naturally, the potential energy landscape is steeper for the fixed case in
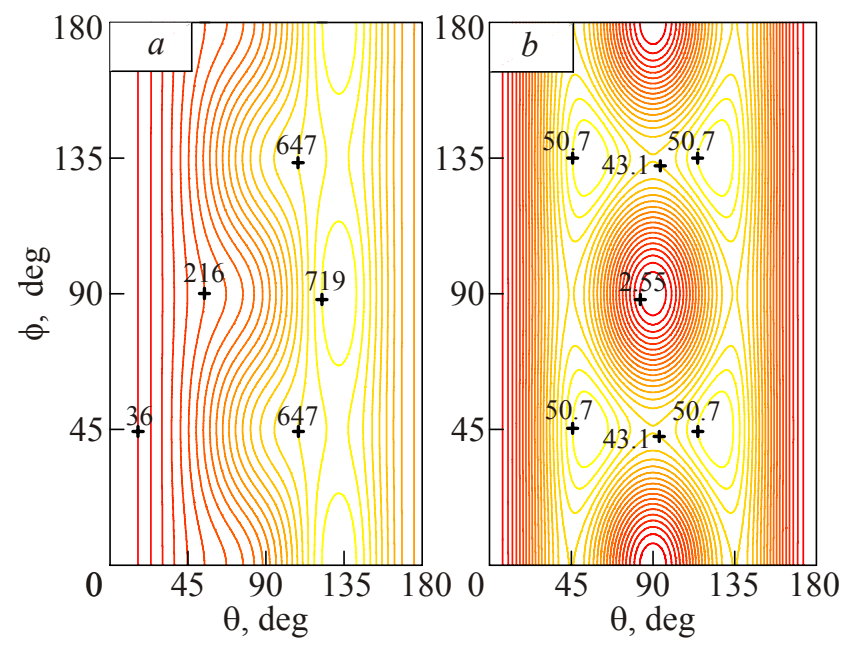

Fig. 2. (Color online) The fixed (a) and adiabatic (b) approaches to compute angular potential energy surfaces for a singlesubstitutional, ground state CO in a fcc Ar lattice. a) Energy minimum is at the $\theta=0$ orientation and rotational barrier reaches $755 \mathrm{~cm}^{-1} . b$ ) The adiabatic surface shows preferable orientations along the crystal $x, y$, and $z$-axis directions. Barrier height is $43.2 \mathrm{~cm}^{-1}$ and the maximum is at $53.3 \mathrm{~cm}^{-1}$. 
Fig. 2,a, which prevents the molecule from reorientational motion.

The adiabatic minima in Fig. 2,b are located in the six $\langle 100\rangle$ crystal axis directions, maxima are in eight $\langle 111\rangle$ directions, and the twelve nearest neighbor orientations $\langle 110\rangle$ locate the saddle points between the adjacent minima. The axis system is such that positive $z$, i.e., $\langle 001\rangle$ corresponds to $\theta=0, x$ axis, i.e., $\langle 100\rangle$ corresponds to $\theta, \phi=90,0$, and $y$ axis, i.e., $\langle 010\rangle$ corresponds to $\theta, \phi=90,90$. The orientational topology differs from that of the Devonshire model for unrelaxed lattice (maxima and saddle points are interchanged), but qualitatively agrees with that obtained by Manz [23], who had 20 and $27 \mathrm{~cm}^{-1}$ saddle point and maximum energies, respectively. With the present potentials $[24,25]$ the barriers appear roughly twice as high.

We collect the lowest state energies in Table 1 for comparison between the two computational approaches. In the fixed lattice approach on row a, the high zero-point energy at $57 \mathrm{~cm}^{-1}$ reflects the angularly squeezed ground-state wave function locked in the $\theta=0$ orientation. More than $55 \mathrm{~cm}^{-1}$ is required for a librational excitation, which indicates a thermally inactive trapping site. Moreover, an increased Boltzmann population in the librational excited states do not lead to a marked spreading of the wave function as the states are still located in the deep well. In the adiabatic approach on row $b$, the three lowest levels are referred to the $n=0$ librational ground state, and the next set of grouped levels close to the barriers compose the $n=1$ excited state $[10,23]$. Inspection of the level structure reveals that the ground state manifold is split into three separate states, each having a different degree of degeneracy. The ground state is at $24.1 \mathrm{~cm}^{-1}$ and is nondegenerate, next three states at $24.9 \mathrm{~cm}^{-1}$ are triple-degenerate and the level at $25.3 \mathrm{~cm}^{-1}$ is double-degenerate. Reducing the lattice constant from 5.31 to $5.26 \AA$ on the $b^{*}$-labeled row of Table 1 probes the sensitivity of the

Table 1. Lowest energy levels (in $\mathrm{cm}^{-1}$ ) for different computational models.

\begin{tabular}{l|c|c|c|c|c|c}
\hline \multicolumn{1}{c|}{ Case } & $E_{1}$ & $E_{2}$ & $E_{3}$ & $E_{4}$ & $E_{5}$ & $E_{6}$ \\
\hline \hline \multicolumn{6}{l}{ Single-substitution site } \\
\hline $\mathrm{a}$ & 57 & 112 & 112 & 160 & 165 & 167 \\
$\mathrm{~b}$ & 24.1 & $24.9(3)$ & $25.3(2)$ & $43.0(3)$ & $44.9(3)$ & $46.9(3)$ \\
$\mathrm{b}^{*}$ & 26.7 & $27.3(3)$ & $27.6(2)$ & $48.1(3)$ & $49.8(3)$ & $51.6(3)$ \\
\hline \multicolumn{7}{l}{ Double-substitution site } \\
\hline OC- $-\times$ & 39 & 69 & 85 & 99 & 114 & 123 \\
$\mathrm{CO}-\times$ & 51 & 84 & 98 & 112 & 129 & 137 \\
\hline
\end{tabular}

Row a: relaxation of lattice structure is assumed only for the $\theta=0$ orientation. Row b: adiabatic lattice response is assumed for all CO orientations. Row $b^{*}$ : contracted lattice. Level degeneracies are indicated for $\mathrm{b}$ and $\mathrm{b}^{*}$ in parentheses. In the doublesubstitution site, the two rows indicate different $\mathrm{CO}$ orientations toward the vacancy $(x)$. result towards thermal expansion. The degeneracy demonstrates the convergence of the gradient descent method in obtaining the fully optimized, adiabatic angular potential that preserves the symmetry. The three levels correlate asymptotically to the $J=0-2$ free-rotor states. Symmetry of the system gives us the possibility of identifying these states with irreducible representations of the $O_{h}$ point group. In ascending energy order, the states can be designated to $A_{1 g}, T_{1 u}$, and $E_{g}$. The crystal field strength is moderate enough to prevent the lowest free-rotor states from coalescing into a single-level ground state as happens in case a and, e.g., in the case of ClF [37] or HArF [38,39] in solid Ar and as was concluded by Anderson and Winn [14] for the present system.

The rovibrational band origin of $2141.6 \mathrm{~cm}^{-1}$ results from the FGH, where the molecule axis was set to one of the equivalent minimum energy directions. The number applies to both approaches above, since also the fixed lattice case was relaxed once for this particular orientation. The vibrational energies can be used to extract the Morse parameters $\omega_{e}=2166.0 \mathrm{~cm}^{-1}$ and $\omega_{e} x_{e}=12.2 \mathrm{~cm}^{-1}$. The matrix shift can be estimated from the empirical gas-phase potential value $2142.9 \mathrm{~cm}^{-1}$. We collect the vibrational parameters obtained for the single-substitution site in Table 2 together with other models to be discussed below.

Table 2. Vibrational data $\left(\mathrm{cm}^{-1}\right)$ obtained by different computational models. The gas phase result refers to that given by the potential $V_{\mathrm{CO}}$, and ab initio results are shifted overall by $-15.8 \mathrm{~cm}^{-1}$

\begin{tabular}{l|c|c|c|c}
\hline \hline Parameter & OC $-\times$ & CO- $\times$ & Single & Gas \\
\hline \hline \multicolumn{5}{l}{ Pair-potential models } \\
\hline$\tilde{v}_{0}$ & 2138.7 & 2140.5 & 2141.6 & 2142.9 \\
$\omega_{e}$ & 2163.7 & 2165.4 & 2166.0 & 2169.8 \\
$\omega_{e} x_{e}$ & 12.5 & 12.4 & 12.2 & 13.5 \\
\hline Ab initio & 2138.8 & 2140.4 & 2140.6 & 2142.9 \\
\hline$\tilde{v}_{0}$ &
\end{tabular}

The obtained level structure for the adiabatic case implies different transitions around the band origin for the Raman and IR spectra, as sketched in Fig. 3, where the $n=0$ manifold is drawn for the lowest vibrational states $v=0$ and 1 (not in scale).

We present the simulated IR and Raman spectra in Fig. 4 using the calculated energy levels and the intensity formula (8). The fixed lattice approach in Fig. 4, $a$ and $c$, i.e., using the potential of Fig. 2, $a$, results in a single peak with no change in intensity with respect to the temperature range applied. However, in the adiabatic approach the librational structure emerges from the spectra as follows. In IR (panel $b$ ), the band is split into two branches for the absorptions $E \leftarrow T$ and $T \leftarrow A$ on the high-energy side of the origin and for $A \leftarrow T$ and $T \leftarrow E$ on the low-energy side. Transitions among the $n=1$ manifold start to emerge 


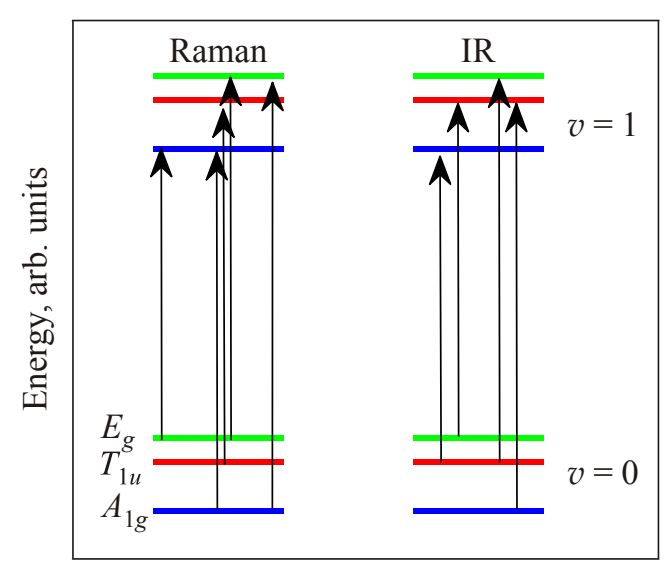

Fig. 3. (Color online) The rovibrational level scheme illustrating the allowed transitions in Raman and IR experiments when the adiabatic, tunneling rotor approach is used to explain the hightemperature spectra. The Raman $A_{1 g} \leftrightarrow A_{1 g}$ component at the $2141.6 \mathrm{~cm}^{-1}$ band origin provides the sharpest signal, while the split transitions are subject to libration-phonon coupling and broadening.

at the elevated temperature further broadening the spectrum. For Raman, the intense, middle peak in Fig. 4, $d$ follows from the ( $Q$ branch) $\Delta J=0$-type transitions among the ground manifold. The contributions can be designated as $A_{1 g} \leftarrow A_{1 g}, T_{1 u} \leftarrow T_{1 u}$, and $E_{g} \leftarrow E_{g}$, transitions. The other lines can be assigned to $A_{1 g} \leftarrow E_{g}$ and $E_{g} \leftarrow A_{1 g}$ transitions $1.25 \mathrm{~cm}^{-1}$ on the red and blues sides of the main peak, respectively, showing the $(0.79+0.46) \mathrm{cm}^{-1}$ level
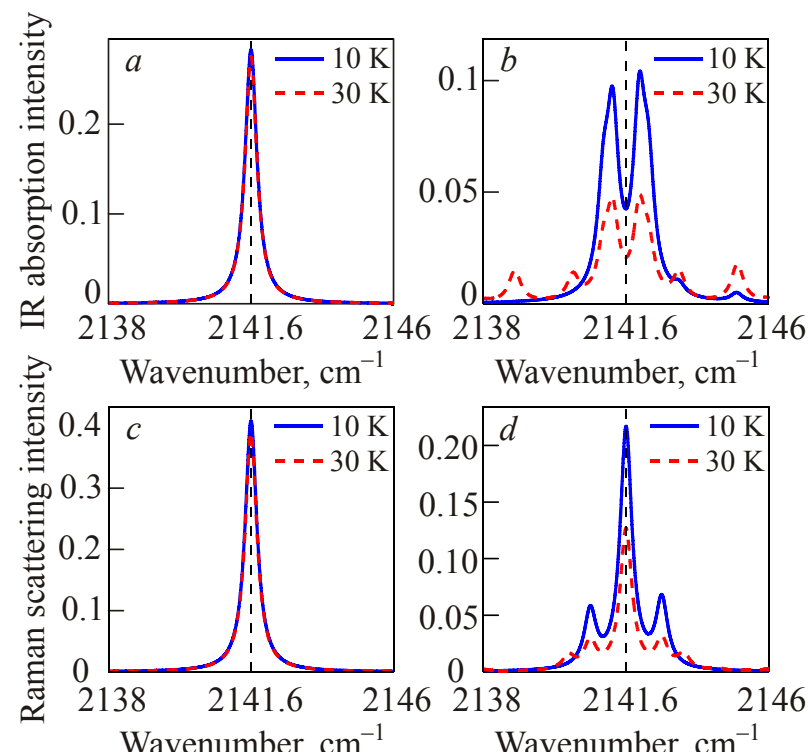

Wavenumber, $\mathrm{cm}^{-1}$

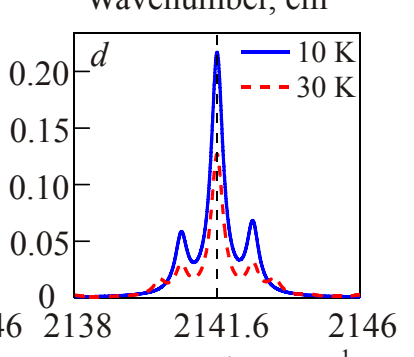

Fig. 4. (Color online) Simulated infrared (panels $a$ and $b$ ) and Raman (panels $c$ and $d$ ) spectra. Left panels refer to the fixedlattice results and right to the fully adiabatic simulations. Temperature variation is shown to affect the line shapes only in the latter case. Vibrational band origin at $\tilde{v}_{0}=2141.6 \mathrm{~cm}^{-1}$ is indicated by dashed line. Rotational constants are 1.92 and $1.24 \mathrm{~cm}^{-1}$ for the fixed and adiabatic cases, respectively. structure of the ground state. The eigenstates are symmetry-adapted pendular hybrid functions of the pure $J$ states which prevents a straightforward branch assignment to $S, Q$, and $R$ or to $\Delta J=0,2$. Transitions among the excited manifold appear weak on both sides of the triplet pattern, as seen in the dashed line. The excited manifold manifests itself on a wider scale (not shown) as a group of transitions blue-shifted by roughly $20 \mathrm{~cm}^{-1}$. This set of transitions originates from the ground levels $n=0$, and was termed the $\Delta n=1$ branch [10,23].

We note that while the temperature samples only ca. $20 \mathrm{~K}$ absolute range, the relative change of temperature is 200 percent. The lattice constant is relatively little affected, and as the consequence so are also the energy levels in the static model. However, besides the thermal expansion, also the dynamics sets forth from what can be considered as a zero-point motion into a random turmoil where static models may not yield the correct description. Therefore the drastic change in viewpoint can be justified. In the warmed lattice we describe by the static crystal field model in effect the averaged potential, where classical rotational diffusion is tantamount to quantum mechanical tunneling effect. In summary, we note that the IR spectrum changes from a single peak, where the sharpness originates from decoupling of the lattice phonons, to a broadened set of transitions, if we treat the cold conditions as the case a locked molecule and change the viewpoint into a thermal average allowing for reorientations at high temperature. The decoupling of lattice phonons at the low temperature is due to the harmonic oscillator-like level structure with large energy gaps. At high temperature, librational level structure with small energy gaps develops, which allows coupling with low-energy phonons. This procedure maintains the sharp spectral line in the case of Raman, since here the rotational selection rules allow the $A_{1 g} \leftrightarrow A_{1 g}$ decoupled transition to exist. While this offers an explanation to the behavior of the peak B, we still need to address the question about the origin of the peak A.

\subsection{Double-substitutional case}

Here we revisit the double-substitutional case examined before by Anderson and Winn [14] to study the effect of different guest-host environments on the spectrum. We did not find a "cluster configuration" as an alternative trapping case identified as the site "A", but discuss the "crystal configuration" in the following. As before, the molecule was found to reside close to one vacant lattice site either the $\mathrm{C}$ or the $\mathrm{O}$ end of the molecule toward the other vacant site. The space between the vacancies was found highly unstable translationally. In the former, global minimum configuration $\mathrm{OC}-\times$, the mass center was displaced from the substitution site only by $0.05 \AA$ toward the other vacancy, while in the $\mathrm{CO}-\times$ configuration the vacancy attraction results to a $0.52 \AA$ shift. Vibrational potential for $\mathrm{CO}$ was then obtained for the optimized structures to solve for 
the red-shifted band origins 2138.7 and $2140.5 \mathrm{~cm}^{-1}$, respectively, which is opposite to the previously obtained order [14]. The corresponding Morse parameters are given in Table 2.

The calculated rotational potential energy is shown in Fig. 5, $a$, which maps the minima at $\theta=90 ; \phi=225,45$. Due to the high barrier between the minima even in the adiabatic case, we consider the two orientations as different trapping sites and issue them with different weight factors. In effect, the results would not differ qualitatively if we used a fixed-lattice approach as in the singlesubstitutional case. The rotational motion is confined to the direction of the vacancy.

The librational energy levels in Table 1 resemble the fixed-lattice result in the single-substitutional case. The energy level difference of $12 \mathrm{~cm}^{-1}$ together with the $19 \mathrm{~K}$ deposition temperature introduce an $\mathrm{OC}$ to $\mathrm{CO}$ probability ratio of 2.5 which is applied in the spectrum simulation. The Raman and IR spectra are presented in Figs. 5,b and $c$,
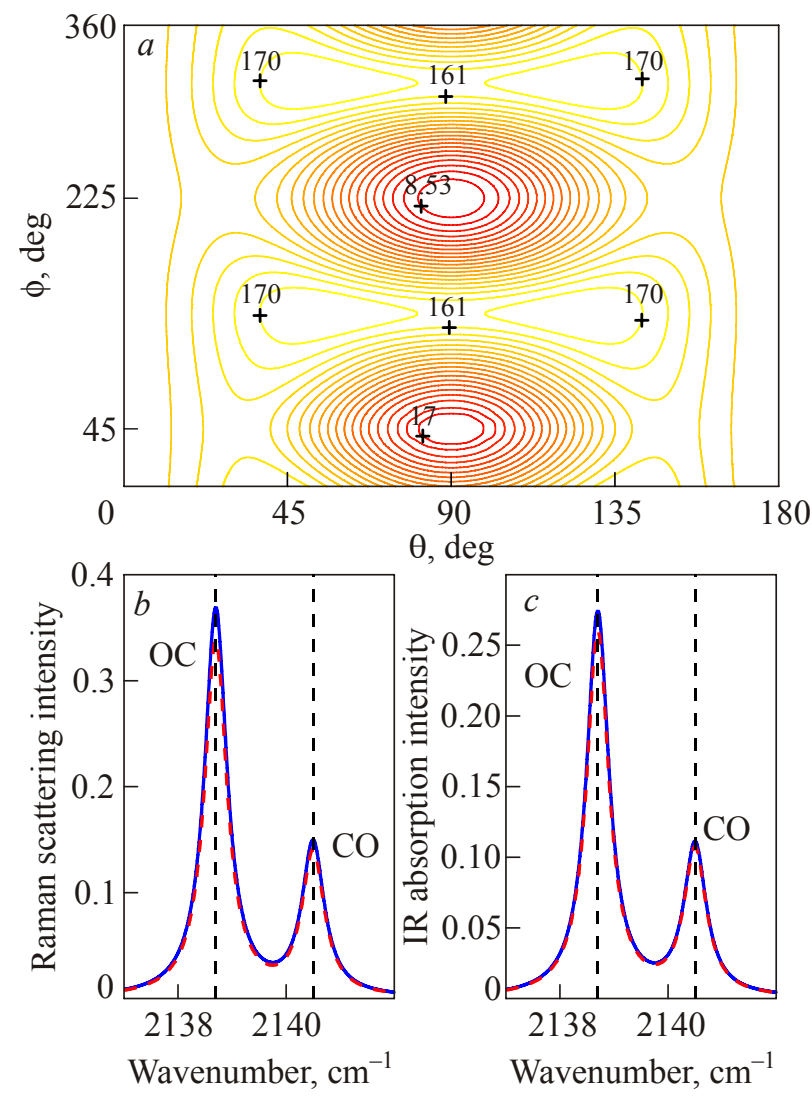

Fig. 5. (Color online) Panel $a$ : The potential energy contour plot for $\mathrm{CO}$ rotation in the adiabatically responsive, double-substitutional cage. Global minimum at $(\theta, \phi)=(90,225)$ corresponds to $\mathrm{CO}$ oriented with carbon end towards the vacancy $(\mathrm{OC}-\times)$. Local minimum at 90,45 with opposite orientation $(\mathrm{CO}-x)$ is $10 \mathrm{~cm}^{-1}$ above. Panels $b$ and $c$ present the simulated Raman and IR spectra that exhibit a doublet structure. Band origins for the global and local minima are shown by broken lines and labeled as $\mathrm{OC}$ and $\mathrm{CO}$, respectively. respectively. Both configurations can be considered as equally stable with respect to thermal excitation. The minimum energy configuration $\mathrm{OC}-x$ gives the most redshifted peak in IR and Raman spectra, and should be more intense by a factor of $5 / 2$ with respect to the other conformation.

The effect of translational motion to the band shape was extracted by additional simulations, where the molecule was moved along the bond axis in both single- and doublesubstitution sites. The resulting line widths (FWHM) were ca. $1.0,2.6$, and $1.5 \mathrm{~cm}^{-1}$ for $\mathrm{OC}, \mathrm{CO}$, and singlesubstitutional cases, respectively. Translational zero-point energies were ca. $15 \mathrm{~cm}^{-1}$ and excitation energies twice as high (nearly harmonic potentials). The adiabatic barrier from OC to CO was $160 \mathrm{~cm}^{-1}$. The results were thus comparable and can not resolve the diverse temperature response in sites A (double) and B (single).

\section{Discussion}

The experimentally observed $\mathrm{CO}$ vibrational transitions in Fig. 1 occur at A) $2136.5 \mathrm{~cm}^{-1}$, B) $2138.3 \mathrm{~cm}^{-1}$, and C) $2139.9 \mathrm{~cm}^{-1}$, thus exhibiting matrix and/or complexation shifts of $6.4,4.6$ and $3.0 \mathrm{~cm}^{-1}$, respectively, from the gas phase. Our hypothesis No. 1 is that the feature called B originates from a single-substitution site. With the model we obtain the band origin at $2141.6 \mathrm{~cm}^{-1}$, which is visible in IR if the fixed lattice (cold) approach is assumed, and emerges in Raman regardless of the approach. We need to shift this transition by $3.3 \mathrm{~cm}^{-1}$ in order to match the experiment. Both fixed and adiabatic approaches are needed to explain the broadening mechanism with respect to temperature, since neither alone with mere librational Boltzmann factors can explain the transition from one peak to a diffuse band in IR and simultaneously maintaining the band shape in Raman. It should be stressed out that incorporation of the two models is not merely a trick to fit the experimental observations. It offers an intuitive and physically sound explanation of the observed line broadening effects and connects the spectral observations to dynamical behavior of the whole guest-host system. The low-temperature limit of a small molecule trapped in a crystal lattice is best described with the static lattice model due to small amplitude motion of the lattice atoms. The sharp lineshape is a manifestation of this. Large energy gaps between the nearly harmonic oscillator librational states provide poor coupling between the molecule and the lattice. As the temperature is raised, amplitude of the lattice atom movement increases providing more space for rotation of the guest molecule. As a result, hindered rotation-like level structure develops that is strongly dependent on the lattice atom coordinates, which naturally explains the strong spectral broadening observed in IR. Despite the modification of the librational level structure, the $Q$ branch remains sharp and shifts only weakly as observed in Raman. An illustrative 
example of this type of behavior is provided by $\mathrm{CN}$ radical isolated in solid xenon, where the $Q$ branch is much sharper than the $P$ and $R$ branches [40,41]. The fundamental difference in the behavior of the IR and Raman spectra can be traced back to the selection rules of vibration-rotation transitions. Therefore, the translation-vibration coupling effect [14] is an unfit explanation since it broadens the Raman transition as well.

The Raman triplet structure in the adiabatic approach is deceivingly similar to the A-B-C pattern, and especially so, if we consider also the $\Delta n=1$ band that could be misinterpreted to the side band D. If the $3.3 \mathrm{~cm}^{-1}$ shift is applied, the shoulder peaks would occur at 2137.1 and $2139.5 \mathrm{~cm}^{-1}$, i.e., the splittings are a bit short from the experimental ones. The splitting is proportional to $B_{\text {eff }}$ and inversely so to the crystal field strength, thus a weaker interaction potential should scale the energetics into the right direction. For the hypothesis sake, however, we must adopt a view, where these Raman side bands are also broadened as happens for the IR branches, and this maintains the central Raman peak as intense as in the $10 \mathrm{~K}$ temperature. The keyword then is the transition selection rule, which in the case of Raman allows the uncoupled states, in particular the $A_{1 g}$ state, to participate in scattering. In IR either the initial or the final state is subject to libration-phonon interaction that is very effective in broadening the transitions as concluded earlier [10,30].

Our hypothesis No. 2 is the coexistence of a doublesubstitution site which yields line positions at 2138.7 and $2140.5 \mathrm{~cm}^{-1}$ for the two orientations $\mathrm{OC}-\times$ and $\mathrm{CO}-\times$, respectively. In order to match with the experiments, a shift of $2.2 \mathrm{~cm}^{-1}$ would result in a doublet at 2136.5 and $2138.3 \mathrm{~cm}^{-1}$. It is tempting to state that the DS doublet accurately reproduces the IR split of $1.8 \mathrm{~cm}^{-1}$. However, the thermal stability and the intensity ratio go against the two peaks being the origin for the doublet A and B structure. Instead, the higher energy contribution can be neglected (i) based on the emergence probability, or (ii) alternatively we can claim that it is hidden below the singlesubstitutional band by coincidence.

The hypotheses 1 and 2 together are supported by the above arguments which resolve previous explanations that are shown contradictory when the new Raman result is taken into account. One wavenumber accuracy is quite an ultimate demand for the series of approximations applied, and absolute wavenumbers are not expected to come out just like in the experiment. Therefore, we must cope with the fact that different scale factors for the single- and double-substitutional results are needed in order to obtain a perfect match with the experiment. To clarify the situation further, we took the optimized structures and applied them to the quantum chemical program, see Table 2 . The wavenumbers turned out as OC- -2 : $2154.6 \mathrm{~cm}^{-1}, \mathrm{CO}-\times$ : $2156.2 \mathrm{~cm}^{-1}$, and single substitution: $2156.4 \mathrm{~cm}^{-1}$ which should be compared to the calculated monomer result of
$2158.7 \mathrm{~cm}^{-1}$ that is in $15.8 \mathrm{~cm}^{-1}$ error as compared to experiment. Scaling with the method error $(2142.9 / 2158.7)$ gives $2138.8,2140.4$, and $2140.6 \mathrm{~cm}^{-1}$ which confirms the hypotheses in two ways. Now the wavenumbers show overlap between the single and the $\mathrm{CO}-\times$ components as discussed above, and additionally reproduces the OC-tosingle or A-B split of $1.8 \mathrm{~cm}^{-1}$. The quantum chemical IR intensities were equal for the two double-substitutional cases (146 and $147 \mathrm{~km} / \mathrm{mol}$ ) and somewhat larger for the single-substitutional case $(163 \mathrm{~km} / \mathrm{mol})$. The same trend was observed for the Raman cross section $(0.98$, $0.99,1.13) \cdot 10^{-14} a_{0}^{2} / \mathrm{sr}$. This discards an argument where the transition dipole moment might be taken more prone to become washed out in $\mathrm{CO}$ orientation than in OC.

For completeness, the issue with dimers needs to be considered. The $\mathrm{CO}$ dimer is known from its nonrigidity and large-amplitude zero-point motion in the gas phase [42]. It exhibits a potential energy surface, where the internal rotation barrier is heavily distance dependent and low enough to support reorientations between the stationary points that are closely separated in energy $[43,44]$. Therefore, we expect that the trapping cage to a large extent determines the conformation in the matrix isolation conditions. Observation of the single frequency for $(\mathrm{CO})_{2}$ indicates either a slipped antiparallel ( $\mathrm{C}$ or O-coordinated) structure, which correspond to the gas phase minimum [43], or a collinear (O-coordinated) structure, which favors the matrix-guided orientation found for the monomer in a double-substitution site. A distorted T-shape estimated earlier as the gas phase minimum structure $[42,45]$ would lead to two IR active vibrational frequencies. Higher polymers should also exhibit separable transitions around the bulk modes 2139 and $2141 \mathrm{~cm}^{-1}[21,46]$.

\section{Conclusions}

We utilized the Raman spectroscopy to unravel the local geometry surrounding the matrix-isolated $\mathrm{CO}$, which acts as the probe to molecule-lattice interaction strength and symmetry. Due to the diatomic simplicity and chemical abundance, the study of CO in Ar has been of fundamental importance in the development of understanding the solid state spectroscopy and reaction dynamics. The temperature dependence of the infrared absorption is an old problem well known to the matrix-isolation community, and the long-standing issue on interpretation has seen phases where the spectral fingerprints have been given assignments to various complexes, librationally broadened rotors, or translationally active oscillators $[10,14,15,23,30]$. Present Raman scattering data has shed new light on the case and called yet for another revision given in this report. The measurement outcome depends on the interplay of temperature and energetics in a manner which may not be as straightforward as previously stated. Vibration, translation, and rotation frequencies of the molecules, the speed 
of which is given by the energy levels for any temperature, are to be contrasted to time scales of the lattice oscillations dictated by the Boltzmann distribution. Thereby the model may need to adapt to a different viewpoint from one temperature regime to another.

We concentrated on the fundamental band of the $\mathrm{CO}$ molecule. The rotational states are found to be quenched into librational ones. While we cannot read the corresponding level structure from any resolved line splittings, the temperature dependence of the spectra provides the key to the interpretation. In particular, the peak called B is put in focus since it exhibits divergent behavior at elevated temperatures when the two spectroscopies are concerned: in IR the peak broadens strongly with increasing temperature while in Raman it remains sharp at all temperatures. We state that the $2138.3 \mathrm{~cm}^{-1}$ peak originates from a singlesubstitution site structure. The interpretation is constructed by the simulations that rely on both fixed and adiabatic lattice equilibrations as the response to the molecular rotational motion. What we have here are static models explaining dynamical, thermally driven phenomena, which can only be good if we are lucky to capture the correct statistical average emerging from the stochastic motion at elevated temperatures.

At the low temperature, the system becomes rather motionless, and the situation is best described with the fixed lattice model. The ground state is that of the aligned superposition of free rotor states yielding the single, sharp-band transition for the Raman and IR alike. This is due to the librational level structure that resembles harmonic oscillator with large energy gaps compared to typical phonon energies. The molecule can be considered to be directionally locked, and sampling of the static, high barriers becomes improbable. In other words, despite being more general, the adiabatic model fails here, since it effectively creates more space around the molecule allowing for reorientations.

At high temperatures, the system behavior changes due to the increasing amplitude of the lattice atoms that opens more space and lowers the barriers for the rotational motion of CO. Under these conditions, the system is more appropriately described with the adiabatic model that results in preserved octahedral symmetry for the trapping site where three nearly degenerate librational states of $A_{1 g}$, $T_{1 u}$, and $E_{g}$ symmetry compose the most occupied ground manifold. While the well-defined transitions between the $A_{1 g}$ are allowed in Raman, the IR case suffers from libration-phonon interaction, since the transitions necessarily concern excited states $T_{1 u}$ and $E_{g}$. The libration-phonon interaction broadens the other Raman lines depicted in Fig. 3 beyond recognition. The same mechanism applies for the computed satellite band ca. $20 \mathrm{~cm}^{-1}$ higher in energy, which refers to genuinely librational, $\Delta n=1$ excitations.
As the main conclusion, the different behavior of the corresponding IR and Raman lines can be attributed to the dynamics of the guest-host system: i) rigid lattice at low temperatures leading to large energy gaps between librational states and overlapping sharp IR and Raman lines; ii) dynamical lattice at high temperatures leading to hindered rotation-like level structure along with the characteristic selection rules for IR and Raman transitions; iii) the strong $Q$ branch in Raman remains sharp while all the other transitions are broadened due to strong coupling with phonons. To obtain overall agreement between experiment and theory, another site of double-substitutional type must be stated as the origin of the thermally stable peak $\mathrm{A}$ at $2136.5 \mathrm{~cm}^{-1}$. The molecule is found to reside in one vacant position and to point with the carbon end toward the other.

As an outlook we note that we agree with the previous assignment of the $2140 \mathrm{~cm}^{-1}$ feature $\mathrm{C}$ to dimers (and the $2149 \mathrm{~cm}^{-1}$ band D to water complexes). However, a photolysis of a dilute $\mathrm{H}_{2} \mathrm{CO}$ precursor, for example, should not produce any $\mathrm{CO}$ polymers. If the peak $\mathrm{C}$ were still present, it must be given another explanation there. This is a topic of our subsequent work.

\section{Acknowledgments}

Dr. Jussi Ahokas is thanked for the assistance in Raman measurements. M. P. and A.O.-M. acknowledge the Emil Aaltonen Foundation for funding. T. K. and J. L. were supported by the Academy of Finland decision No. 124974.

1. L. Khriachtchev, A. Lignell, J. Juselius, M. Räsänen, and E. Savchenko, J. Chem. Phys. 122, 014510 (2005).

2. L. Pauling, Phys. Rev. 36, 430 (1930).

3. M.E. Fajardo, C.M. Lindsay, and T. Momose, J. Chem. Phys. 130, 244508 (2009).

4. A.G. Maki, J. Chem. Phys. 35, 931 (1961).

5. H. Vu. M.R. Atwood, and B. Vodar, J. Chem. Phys. 38, 2671 (1963).

6. G.E. Leroi, G.E. Ewing, and G.C. Pimentel, J. Chem. Phys. 40, 2298 (1964).

7. J.B. Davies and H.E. Hallam, J. Chem. Soc., Faraday Trans. 2 68, 509 (1972).

8. H. Dubost and L. Abouaf-Marguin, Chem. Phys. Lett. 17, 269 (1972).

9. H. Dubost, Chem. Phys. 12, 139 (1976).

10. H. Dubost, A. Lecuyer, and R. Charneau, Chem. Phys. Lett. 66, 191 (1979).

11. G.J. Jiang, W.B. Person, and K.G. Brown, J. Chem. Phys. 62, 1201 (1975).

12. Y. Ogawara, A. Bruneau, and T. Kimura, Anal. Chem. 66, 4354 (1994).

13. H.S. Han and K. Kim, J. Mol. Struct.: THEOCHEM 418, 1 (1997).

14. D.T. Anderson and J.S. Winn, J. Phys. Chem. A104, 3472 (2000). 
15. H. Abe, H. Takeo, and K.M.T. Yamada, Chem. Phys. Lett. 311, 153 (1999).

16. H. Abe and K.M.T. Yamada, J. Chem. Phys. 114, 6134 (2001).

17. H. Abe and K.M.T. Yamada, J. Chem. Phys. 121, 7803 (2004).

18. F. Thibault, R.Z. Martinez, J.L. Domenech, D. Bermejo, and J.-P. Bouanich, J. Chem. Phys. 117, 2523 (2002).

19. H. Abe and K.M. T. Yamada, Struct. Chem. 14, 211 (2003).

20. A. Olbert-Majkut, J. Ahokas, J. Lundell, and M. Pettersson, Chem. Phys. Lett. 468, 176 (2009).

21. Vadim G. Manzhelii and Yuri A. Freiman, Physics of Cryo-crystals, AIP Press, Woodbury (1997).

22. A.F. Devonshire, Proc. R. Soc. Lond. A153, 601 (1936).

23. J. Manz, J. Am. Chem. Soc. 102, 1801 (1980).

24. K.T. Tang and J.P. Toennies, J. Chem. Phys. 118, 4976 (2003).

25. T.B. Pedersen, J.L. Cacheiro, B. Fernández, and H. Koch, J. Chem. Phys. 117, 6562 (2002).

26. P. Huxley and J.N. Murrel, J. Chem. Soc., Faraday Trans. 2 79, 323 (1983).

27. R.N. Zare, Angular Momentum, Wiley, New York (1988).

28. A.J. Stone, The Theory of Intermolecular Forces, Clarendon Press, Oxford (1996).

29. P.J. Davis and P. Rabinowitz, Methods of Numerical Integration, Academic Press, New York (1975).

30. R.B. Gerber, M. Berkowitz, and V. Yakhot, Mol. Phys. 36, 355 (1978).

31. D.T. Colbert and W.H. Miller, J. Chem. Phys. 96, 1982 (1992).

32. F. Weigend and M. Häser, Theor. Chem. Acc. 97, 331 (1997).

33. F. Weigend, M. Häser, H. Patzelt, and R. Ahlrichs, Chem. Phys. Lett. 294, 143 (1998).
34. C. Hättig, Phys. Chem. Chem. Phys. 7, 59 (2005).

35. F. Weigend and R. Ahlrichs, Phys. Chem. Chem. Phys. 7, 3297 (2005).

36. TURBOMOLE V6.1 2009, a development of University of Karlsruhe and Forschungszentrum Karlsruhe $\mathrm{GmbH}$, 1989-2007, TURBOMOLE GmbH, since 2007; available from http://www.turbomole.com.

37. T. Kiljunen, M. Bargheer, M. Gühr, N. Schwentner, and B. Schmidt, Phys. Chem. Chem. Phys. 6, 2932 (2004).

38. A.V. Bochenkova, L. Khriachtchev, A. Lignell, M. Räsänen, H. Lignell, A.A. Granovsky, and A.V. Nemukhin, Phys. Rev. B77, 094301 (2008).

39. A.V. Bochenkova, V.E. Bochenkov, and L. Khriachtchev, J. Phys. Chem. A113, 7654 (2009).

40. A. Thoma, G. Schallmoser, A.M. Smith, B.E. Wurfel, and V. Bondybey, J. Chem. Phys. 100, 5387 (1994).

41. M. Pettersson, J. Lundell, L. Khriachtchev, and M. Räsänen, J. Chem. Phys. 109, 618 (1998).

42. A.W. Meredith and A.J. Stone, J. Phys. Chem. A102, 434 (1998).

43. L.A. Surin, D.N. Fourzikov, T.F. Giesen, S. Schlemmer, G. Winnewisser, V.A. Panfilov, B.S. Dumesh, G.W.M. Vissers, and A. van der Avoird, J. Phys. Chem. A111, 12238 (2007).

44. K.M.T. Yamada, J. Mol. Spectrosc. 254, 87 (2009).

45. L.M. Nxumalo, E.K. Ngidi, and T.A. Ford, J. Mol. Struct. 786, 168 (2006).

46. G. Firanescu and R. Signorell, J. Phys. Chem. B113, 6366 (2009). 